\title{
Dinámica de conservación en el lugar de las variedades criollas de maíz en la aldea EI Trapiche, Choluteca, Honduras
}

Iris Rodríguez y Yensi Flores ${ }^{1}$

Jorge Carrasco ${ }^{2}$

\section{RESUMEN}

La diversidad y la conservación de los cultivos tradicionales han sido poco estudiadas, por eso el objetivo de este estudio es describir la dinámica de conservación en el lugar del cultivo de maíz en una aldea rural de Honduras, a través del análisis de las prácticas de manejo y la caracterización morfológica de las variedades criollas de maíz que son utilizadas en esta localidad, lo cual permite evaluar algunas de las características claves en su identificación.

Este trabajo se llevó a cabo en cuatro caseríos pertenecientes a la aldea El Trapiche, municipio de Choluteca, en la región sur de Honduras. Se consultó a través de una encuesta a 26 cultivadores de maíz de esta zona sobre las características que les permiten identificar las variedades criollas de maíz que cultivan, prácticas agrícolas y el intercambio de material de siembra. Además, se realizó una caracterización morfológica de las variedades criollas que cultivan mediante la medición de caracteres morfológicos de planta y mazorca.

Los resultados demuestran cómo las actividades de manejo tradicional originan un proceso dinámico de conservación en el sitio a través del tiempo para el germoplasma de maíz cultivado. Los agricultores reportaron siete variedades: cinco criollas, una variedad foránea y la variedad comercial donada por el Gobierno de Honduras. Las variedades identificadas fueron: maicito, amarillo, planta baja, cuarenteño, negro y maizón; esta última considerada una variedad foránea y mejorada. La mayoría de ellas son de grano blanco y destinada para la alimentación humana, la variedad amarillo es utilizada principalmente para la alimentación animal. La estrategia de siembra

\footnotetext{
${ }^{1}$ Beneficiarias de una beca sustantiva de la DICYP, profesoras investigadoras de la Escuela de Biología, Facultad de Ciencias, UNAH: iris.rodriguez@unah.edu.hn; yensiflores@gmail.com 2 Profesor investigador de la Escuela de Microbiología, Facultad de Ciencias, UNAH: jcarrasco60@yahoo.es
} 
es a bordón, no se reporta uso de trabajo animal, el $50 \%$ de los encuestados manifiestan el uso de gramoxone para la limpieza del terreno; mientras que el $38.5 \%$ indican que usan urea para fertilizar el suelo. Con respecto al intercambio de semilla para siembra, el $53 \%$ de los encuestados notifica el intercambio de material a través de procesos de compra y regalo de familiares y amigos. Es evidente la persistencia de un sistema tradicional en el cultivo del maíz, lo que se observó en sus prácticas y el uso de semillas locales adaptadas a suelos pedregosos que dificulta la modernización agrícola y el uso de semillas mejoradas en esta zona.

Palabras clave: dinámica de conservación, maíz, variedad criolla, manejo tradicional.

\section{ABSTRACT}

Diversity and conservation of traditional crops have been scarcely studied. The objective of this study is to describe the in situ conservation dynamics of corn crops in a rural village of Honduras, through the analysis of management practices and the morphological characterization of native corn varieties that are being used at this location, which permits the evaluation of key characteristics for its identification.

This work was carried out in four communities in the village of El Trapiche, Choluteca in southern Honduras. Consultations were conducted through a survey of 26 corn growers in the area, about the characteristics that allow them to identify the cultivated varieties of corn, agricultural practices and planting material exchanges. We also carried out a preliminary assessment of the varieties they grow through the measurements in situ of morphological characteristics of the plant and corncob.

The results demonstrate how traditional management creates an overtime dynamic process in situ for grown corn germplasm. Farmers reported seven identified varieties, five native and one foreign, and the commercial variety donated by the Honduran government. The identified varieties were: young corn, yellow, ground, cuarenteño, black and maizón (considered a foreign and improved variety).

Most of the varieties are white grain, which is intended for human consumption, the yellow variety is used mainly for animal feed. The planting strategy reports an absence of animal labor, $50 \%$ of respondents report using Gramoxone for land clearing and $38.5 \%$ of respondents indicate the use urea as soil fertilizer. Regarding the exchange of sowing seeds, $53 \%$ of respondents report sharing process material 
through purchase and gifts from family and friends. Clearly the persistence of a traditional corn can be observed in their practices and the use of local seeds that has been adapted to rocky soils which hinders agricultural modernization and the use of improved seeds in the area.

Keywords: maize, conservation dynamics, native variety, traditional management 


\section{INTRODUCCIÓN}

En América Latina, los agricultores siguen cultivando sus variedades criollas de maíz y el uso de germoplasma mejorado está concentrado en zonas en donde el maíz es un cultivo comercial (Ferro y otros, 2008). La dinámica en el manejo de recursos locales que los campesinos desarrollan en sus parcelas, ha ganado interés por sus posibles implicaciones en la conservación en el lugar de la diversidad cultivada.

Las prácticas de cultivo, que incluyen en muchas ocasiones el manejo de numerosas poblaciones diferentes de maíz en un área pequeña, ha propiciado toda la variabilidad que existe en los sistemas de fincas tradicionales (Jarvis y otros, 1999; Fernández y otros, 2010). Por lo anterior, es importante conocer cómo se dan estos procesos en comunidades rurales en donde los campesinos aún practican formas tradicionales de cultivo, con un predominio del trabajo humano y animal, gran variedad de cultivos, múltiples variedades de cada cultivo y mayor uso de variedades adaptadas localmente (Tuxill y Nabhan, 2001).

Desde épocas ancestrales hasta la actualidad, el maíz es el eje de nuestra alimentación, ya que su origen y evolución bajo domesticación se inició en Mesoamérica y continúa hoy en día en la milpa tradicional (Hernández, 1985). La diversidad maicera está relacionada con la persistencia cultural, los factores bióticos y abióticos tales como suelo, clima y sistemas de cultivo tradicionales manejados por los milperos locales.

Los agricultores toman decisiones concernientes a la siembra, manejo y cosecha, además seleccionan plantas de maíz con las características morfológicas preferidas; la mayoría de las veces sembrando numerosas poblaciones en una misma área, lo cual origina un proceso dinámico de conservación en ese lugar que perdura hasta este tiempo (Hernández, 1993).

En consecuencia, el objetivo de este estudio es describir la dinámica de conservación en el lugar del cultivo de maíz en una aldea rural de Honduras, a través del análisis de las prácticas de manejo y la caracterización morfológica de las variedades criollas de maíz que son utilizadas en esta localidad, lo cual permite evaluar algunas de las características claves en su identificación. 


\section{METODOLOGÍA}

\section{Área de estudio}

Este estudio se llevó a cabo en cuatro caseríos pertenecientes a la aldea El Trapiche, del municipio de Choluteca, departamento de Choluteca, ubicado en la región sur de Honduras. Las comunidades visitadas fueron: El Trapiche, La Trinidad, La Puerta y El Capulín (ver figura 1). La aldea El Trapiche tiene un área de aproximadamente 34.14 $\mathrm{km}^{2}$, a una altura entre 200-500 msnm. Su topografía es irregular, constando básicamente de laderas con pendientes altas. El suelo es pedregoso, erosionado, áspero y seco, perteneciente a la categoría VII según las categorías de capacidad agroecológica (Simmons, 1959).

Figura 1. Mapa del área de estudio de la aldea "El Trapiche"

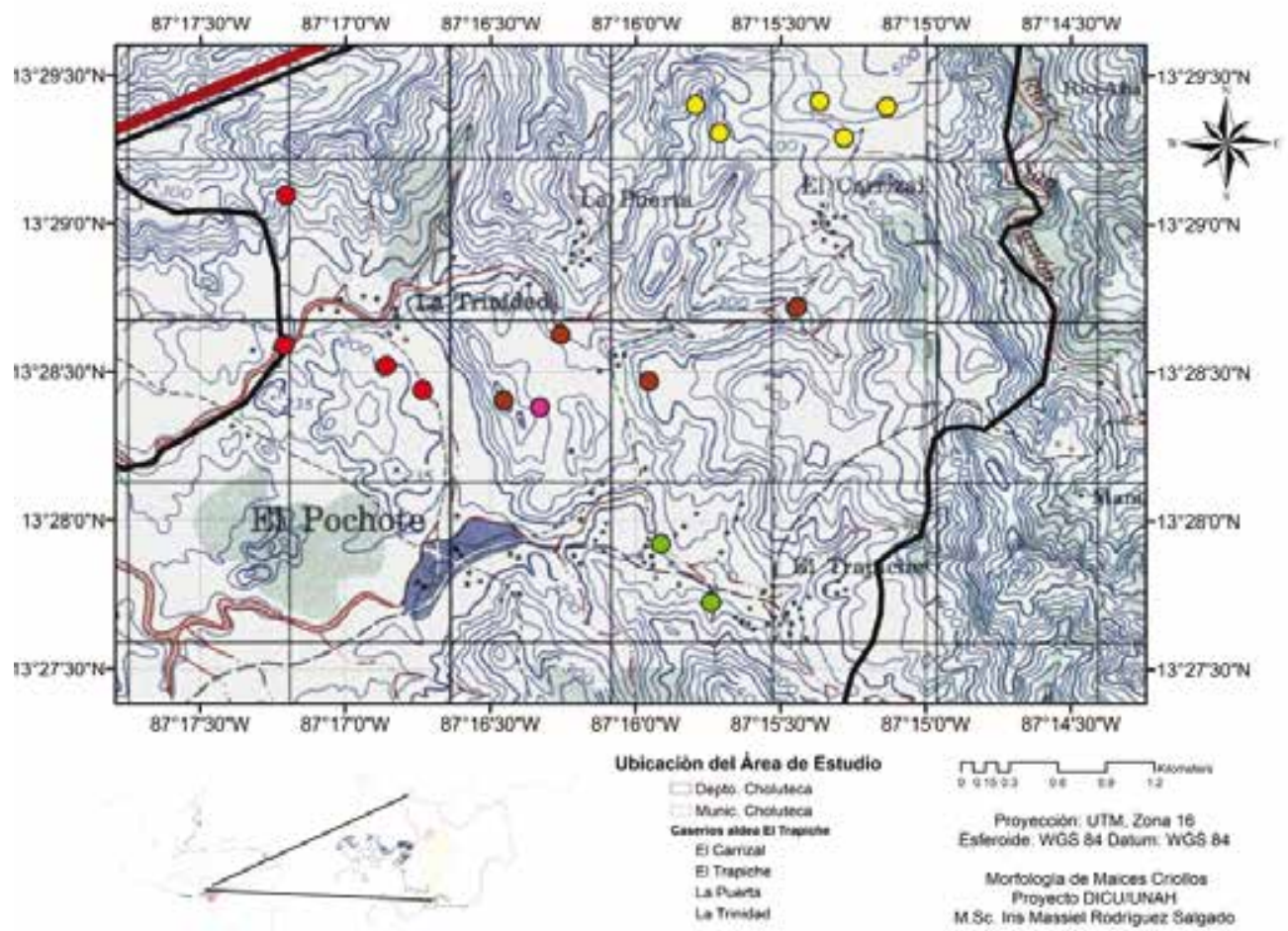

Las condiciones climáticas son las regidas por las condiciones de la zona de vida del bosque seco tropical, cuya característica principal es presentar dos estaciones marcadas, la seca con duración promedio de siete meses y la lluviosa entre los meses de mayo a agosto, con un veranillo intermedio entre los meses de julio y agosto, presentando una pluviosidad de 0 a $64 \mathrm{~mm}$ en la época seca y de 100 
a $340 \mathrm{~mm}$ en la estación lluviosa. Las actividades económicas que fueron reportadas para esta aldea son la agricultura y ganadería (Toledo, 1990).

\section{Obtención y medición de datos}

El estudio se realizó con la colaboración de 26 agricultores de la aldea y se visitaron las parcelas de cultivo de maíz pertenecientes a estos agricultores. El muestreo se realizó entre los meses de septiembre a diciembre del año 2008, durante el ciclo productivo de postrera. Se colectaron datos usando las siguientes técnicas:

1. Aplicación de encuesta y observación de prácticas de siembra, manejo de cultivo y de semilla. Se aplicó a los agricultores una encuesta semiestructurada, se registró el nombre, edad, sexo y ocupación de cada uno de los encuestados, también se indagó sobre las estrategias de manejo proporcionado al cultivo, tales como propósito del cultivo, prácticas de siembra, cantidad sembrada y manejo de plagas asociadas. Asimismo, se hicieron preguntas acerca del manejo de las semillas cultivadas para conocer su origen, actividad de intercambio, preferencia, caracteres diferenciadores de las variedades y su conservación.

2. Medición en el lugar del cultivo de caracteres morfológicos de planta y mazorca. La medición de caracteres de la planta se llevó a cabo en los meses de septiembre y octubre. Para cada parcela muestreada se incluyó de 20 a 30 individuos de cada parcela. Para asegurar la muestra se marcaron dos transeptos lineales cruzados de $20 \mathrm{~m}$ de longitud, tomando la información de las plantas que caían en los transeptos. Para cada parcela de cultivo se tomaron los siguientes datos: coordenadas geográficas de ubicación, altitud (msnm), tipo de terreno, técnica de siembra, tipo de suelo, tipo de vegetación circundante, otros cultivos, tamaño aproximado de parcela en metros cuadrados, presencia de plagas.

La medición de los caracteres morfológicos de la planta se llevó a cabo posteriormente al periodo de floración de las plantas, asegurando la madurez completa de los caracteres vegetativos. Los transeptos fueron demarcados en el centro de la parcela de cultivo para evitar el efecto de borde. Se marcaron a las plantas incluidas en cada transepto con un número único y se procedió a medir los siguientes caracteres vegetativos: altura de planta y altura de mazorca. Las mazorcas de las plantas señaladas fueron recolectadas y trasladadas al Laboratorio de Histología Vegetal y Etnobotánica SL-W del Departamento de Biología, en donde fueron almacenadas y se realizaron las siguientes mediciones de la mazorca: longitud y número de granos por hilera. 


\section{ANÁLISIS DE DATOS}

Los datos de la encuesta fueron tabulados en una hoja de Excel 2010, se hizo un análisis de los factores socioculturales y antropológicos que determinan la dinámica del manejo de siembra, cultivo y semilla a través de un análisis de frecuencias y porcentajes.

Los datos obtenidos en la caracterización morfológica de la planta y la mazorca también fueron tabulados en una hoja de Excel y analizados con el programa estadístico Minitab 16, utilizando los parámetros de la estadística descriptiva, tales como medida de tendencia central, medidas de dispersión y distribución de frecuencias.

\section{RESULTADOS}

Todas las personas encuestadas fueron del sexo masculino, agricultores de oficio, con una edad promedio de 48 años. Las 26 parcelas que fueron evaluadas pertenecen a estos agricultores, de las cuales el $93 \%$ se ubican en la zona agrícola, situada en laderas de pendiente alta que bordean a los caseríos.

Domina la mano de obra familiar, poca tecnificación de cultivos y la producción destinada mayormente al autoconsumo. Las parcelas de cultivo presentan un tamaño que varió entre 0.7- 2.9 ha, con un promedio de 1.55 ha. El $71 \%$ (19/26) de los productores tienen dos ciclos productivos, el de primera que se da con el inicio de la estación lluviosa en la zona sur de Honduras, en el mes de abril y el ciclo de postrera, que inicia en el mes de agosto, correspondiente a la reanudación de la estación lluviosa después del periodo de canícula.

La dinámica de conservación llevada a cabo por los agricultores se puede determinar con base en las prácticas de siembra y manejo del cultivo, origen e intercambio de semillas y la utilización de variedades locales cultivadas.

Prácticas de siembra y manejo del cultivo

Para evaluar el manejo del cultivo de maíz, se consultó a los entrevistados con respecto a la técnica de siembra, preparación de suelo y uso de agroquímicos. Los 
agricultores reportaron metodologías de siembra convencionales, colocando de 3 a 4 semillas por agujero con una distancia promedio de $60 \mathrm{~cm}$, sin reportar el uso de trabajo animal. Todos reportaron una previa preparación del suelo mediante limpieza y deshierbe, el $50 \%$ (13/26) informa el uso del herbicida gramoxone para la limpieza del terreno y el $38.5 \%$ (10/26) señala el uso de urea para fertilizar el suelo. También se observó el mantenimiento de policultivo, en cinco de las parcelas estudiadas, en donde del maíz se acompañó con maicillo (Sorghum bicolor) y ocasionalmente con frijol (Phaseolus vulgaris).

También se observó que los agricultores mezclan las variedades cultivadas dentro de la misma parcela y hasta en el mismo surco de siembra. Adicionalmente, se registró que el periodo de desarrollo de las plantas muestreadas de las diferentes variedades, es el mismo o muy similar, por lo que son tratados como una sola población. Sin embargo, al momento de recolección de los granos, ellos los clasifican según sus características más diferenciadoras, tales como color de grano, y almacenan las semillas de las mejores mazorcas de cada variedad para el cultivo del siguiente ciclo.

Adicionalmente, ellos expresaron que la selección de las variedades a cultivar en el ciclo de siembra está íntimamente ligado a las condiciones climáticas de ese momento, pues al tener mejores inviernos pueden sembrar variedades de mayor rendimiento; pero, en el caso de inviernos cortos y veranos muy secos, se ven obligados a utilizar variedades de ciclos muy cortos, con menores tasas de rendimiento.

\section{Origen e intercambio de semillas}

Las variedades criollas reportadas en este estudio son adquiridas por medio de la herencia de las semillas de una generación a otra. Los agricultores entrevistados afirmaron haber obtenido sus primeras semillas como herencia y haberlas cultivado durante toda su vida. Ellos indicaron que la principal razón para el sustento persistente de estas variedades, se debe a su alta adaptación a las condiciones climáticas de la zona; específicamente, su resistencia a la sequía y capacidad de crecimiento en suelo árido, no apto para el cultivo. Sin embargo, expresaron que estas variedades presentan bajos niveles productivos, lo que se reflejó en el tamaño de la mazorca y la cantidad de granos recuperados en cada una. Como consecuencia, los productores de la zona, han introducido algunas variedades foráneas de mayor rendimiento, para el mejoramiento de la productividad de sus variedades criollas.

Igualmente se documentó el intercambio de semilla entre agricultores de diferentes 
caseríos, a través de la forma de obtención de las semillas y el intercambio intencional de semillas con otros agricultores. Se consultó a cada agricultor cómo consigue cada una de las semillas de los materiales que siembra, los resultados se reflejan en la figura 2, en donde se indica que aunque los agricultores obtienen sus semillas de cosechas anteriores, estos adquieren semillas de otras parcelas, pudiendo ser estas de las mismas o diferentes variedades para la introducción de características de interés a su cultivo.

Figura 2. Origen de las semillas sembrada por los agricultores en El Trapiche

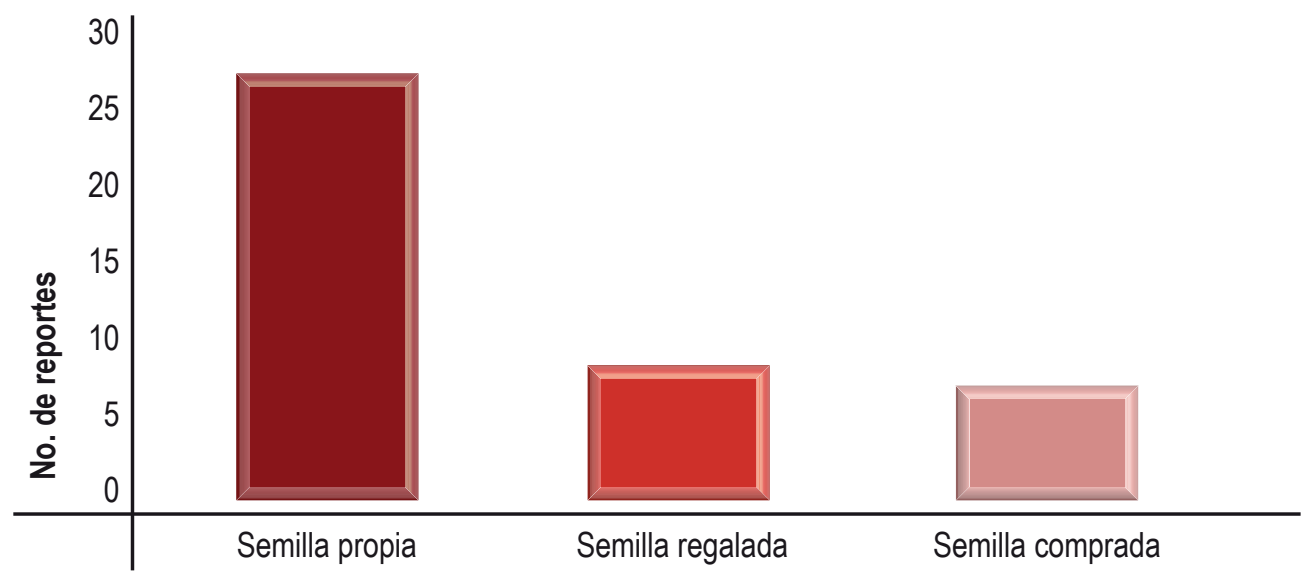

Según el análisis realizado, el $62.2 \%$ (16/26) de los entrevistados indican que la forma tradicional de obtención de semilla es a partir de la cosecha previa, mediante la selección y almacenamiento de los mejores materiales.

La incorporación de nuevo material para siembra se evidencia a través del proceso de compra y regalo de semillas entre los miembros de la comunidad. También se indagó con cada agricultor si hay intercambio intencional de semilla para siembra, aproximadamente el $73 \%$ (19/26) respondió que sí, un $15 \%$ (4/26) dice que no lo hace y el $12 \%(3 / 26)$ no sabe o no responde.

\section{Variedades cultivadas}

En este trabajo de investigación se consideró el concepto "variedad", utilizado por los campesinos, definida como el conjunto de lotes de semilla que llevan el mismo nombre y valorado como un conjunto de granos de maíz de un mismo tipo, 
seleccionado para ser sembrados durante un ciclo de cultivo y la progenie directa de estas semillas (Louette, 1996).

Considerando la definición previa, se determinó que en la aldea El Trapiche fueron reportadas siete variedades: cinco criollas, una foránea y una comercial. Las variedades criollas mencionadas son: maicito, amarillo, planta baja, cuarenteño y negro; la variedad foránea es denominada maizón y la variedad comercial, donada por el Gobierno, se conoce como semilla de bono agrícola. La mayoría de las variedades son de grano blanco y se destinan a la alimentación humana, mientras que la variedad de grano amarillo es utilizada principalmente para la alimentación animal.

De las siete variedades mencionadas en las encuestas, cuatro fueron encontradas durante el muestreo para caracterización. El maicito es la variedad predominante, encontrado en 18 de las parcelas muestreadas. Le sigue a este, el maíz amarillo y planta baja, ambos encontrados en tres parcelas. La variedad foránea, el maizón, se encontró en una sola parcela. El $30 \%(\mathrm{~N}=26)$ de las parcelas muestreadas presentaban áreas de cultivo en las que se sembró más de una variedad, combinando maicito con planta baja o amarillo.

Los agricultores establecen diferencias entre las variedades que cultivan, el maicito es identificado principalmente por ser una planta alta, con tuza morada, mazorca pequeña de granos blancos y planos, dispuestos de forma regular. La variedad planta baja fue identificada por ser una planta de baja estatura, mazorca pequeña de forma redonda, con granos blancos, redondos y de disposición regular. La variedad amarillo fue identificada por ser una planta de baja estatura, de mazorca larga con granos amarillos, blancos, rojos, anaranjados y combinaciones de amarillo y blanco, dispuestos en espiral. La variedad maizón fue caracterizada por ser la planta de menor estatura, de granos blancos, con mazorca de mayor longitud y cobertura de granos.

Los resultados obtenidos en la caracterización morfológica de las variedades encontradas presentaron, en su mayoría, resultados homogéneos entre variedades y alta variabilidad intravarietal, denotando un continuo morfológico (ver cuadros 1 y 2).

En cuanto a los resultados alcanzados en la medición de caracteres, la planta y de mazorca de las cuatro variedades estudiadas, muestra que el maíz amarillo presenta los valores más bajos en todos los caracteres evaluados; mientras que el maicito muestra valores más altos para caracteres vegetativos e intermedios en mazorca. Por último, la variedad planta baja, aunque presenta resultados intermedios 
en caracteres vegetativos, exhibe los valores más altos para caracteres de mazorca entre las variedades criollas. La variedad foránea, el maizón, similar a la variedad criolla planta baja, presentó los resultados más bajos en caracteres vegetativos y los más altos en caracteres de mazorca.

Cuadro 1. Características vegetativas de las variedades estudiadas en las 26 parcelas muestreadas

\begin{tabular}{|c|c|c|c|c|c|}
\hline Variedad & & Altura & $\begin{array}{l}\text { Altura } \\
\text { (cm) }\end{array}$ & mazorca & $\begin{array}{l}\text { No. } \\
\text { hojas }\end{array}$ \\
\hline Maicito & $\begin{array}{l}(\bar{x}) \\
\delta\end{array}$ & $\begin{array}{l}138.61 \\
46.45\end{array}$ & $\begin{array}{l}50.36 \\
42.00\end{array}$ & 4 & $\begin{array}{l}8.60 \\
3.05\end{array}$ \\
\hline Planta baja & $\begin{array}{l}(\bar{x}) \\
\delta\end{array}$ & $\begin{array}{l}119.46 \\
57.00\end{array}$ & $\begin{array}{l}37.30 \\
46.57\end{array}$ & & $\begin{array}{l}7.38 \\
2.88\end{array}$ \\
\hline Amarillo & $\begin{array}{l}(\bar{x}) \\
\delta\end{array}$ & $\begin{array}{l}116.94 \\
30.87\end{array}$ & $\begin{array}{l}26.11 \\
26.67\end{array}$ & & $\begin{array}{l}7.01 \\
1.99\end{array}$ \\
\hline Maizón & $\begin{array}{l}(\bar{x}) \\
\delta\end{array}$ & $\begin{array}{l}108.61 \\
29.18\end{array}$ & $\begin{array}{l}13.69 \\
1.14\end{array}$ & & $\begin{array}{l}5.65 \\
1.37\end{array}$ \\
\hline
\end{tabular}

Cuadro 2. Características de mazorca de las variedades estudiadas en las 26 parcelas muestreadas

\begin{tabular}{|c|c|c|c|c|}
\hline Variedad & \multicolumn{2}{|c|}{ Longitud mazorca (cm) } & \multicolumn{2}{|c|}{ No. de granos por hilera } \\
\hline Amarillo & $\begin{array}{l}(\bar{x}) \\
\delta\end{array}$ & $\begin{array}{l}11.08 \\
3.18\end{array}$ & $\begin{array}{l}19.89 \\
8.81\end{array}$ & \\
\hline Maicito & $\begin{array}{l}(\bar{x}) \\
\delta\end{array}$ & $\begin{array}{l}12.00 \\
3.05\end{array}$ & $\begin{array}{l}21.32 \\
8.43\end{array}$ & \\
\hline Planta baja & & $\begin{array}{l}11.98 \\
2.74\end{array}$ & $\begin{array}{l}22.57 \\
8.18\end{array}$ & \\
\hline Maizón & & $\begin{array}{l}13.58 \\
2.68\end{array}$ & $\begin{array}{l}26.17 \\
7.41\end{array}$ & \\
\hline
\end{tabular}




\section{DISCUSIÓN}

Es evidente la persistencia de un sistema tradicional de cultivo del maíz en esta aldea; el uso exclusivo de trabajo humano, la poca utilización de agroquímicos y el mantenimiento de diversas variedades lo corrobora. Las variedades identificadas se encuentran bien adaptadas a las condiciones ambientales locales, acondicionadas a suelos pedregosos que dificultan la modernización agrícola y a condiciones de sequía y altas temperatura, por lo que probablemente las variedades mejoradas no pueden ser utilizadas.

Las condiciones climáticas y de suelo ejercen una gran presión sobre las variedades cultivadas en la zona. La dinámica de conservación de las variedades criollas en la aldea El Trapiche es el producto de la capacidad de adaptación de los maíces cultivados y el rendimiento esperado de los cultivos. Esta dinámica se presenta desde el proceso de obtención y selección de la variedad a cultivar en el ciclo productivo, obligando a los campesinos a mantener una amplia gama de variedades, hasta en la introducción de variedades foráneas para perpetuar características favorables en sus variedades criollas, como parte de un proceso de mejoramiento de su semilla para aumentar su rendimiento.

Esta dinámica fue observada y confirmada por los agricultores de la zona, pero aclaran que las variedades (maicito, el cuarenteño y amarillo) se utilizan primordialmente por su resistencia a la sequía, aunque sus rendimientos sean menores, pero en condiciones de sequía no asumirán el riesgo de la pérdida del cultivo y siembran variedades con mayor rendimiento.

El flujo genético de variedades foráneas que han sido introducidas para mejoramiento del rendimiento de las variedades locales, puede ser deducido a partir de los datos de las entrevistas y las observaciones hechas en las características morfológicas de las variedades reportadas. En tal sentido, se puede asumir que dos de las variedades que utilizan actualmente y que nombran como locales o propias de la región, son probablemente producto del cruce de variedades introducidas y el maicito.

Dentro de estas, la variedad planta baja, se encuentra en la aldea recientemente, muy pocos de los campesinos reportaron tenerlo o cultivarlo, adicionalmente, este presenta características similares a las del maicito, como la forma del grano, forma de mazorca, color de tallo y las diferencias esenciales son aquellas que aumentan su rendimiento, menor tamaño de planta, mayor tamaño de mazorca y mayor número de 
granos. Adicionalmente, la homogeneidad obtenida en la expresión de sus caracteres fenotípicos indica que probablemente este es producto de cruce con un maíz mejorado.

La dinámica observada para la variedad amarillo, es compleja, pues muestra que fue introducida a la aldea hace aproximadamente 100 años, por lo que ya es una variedad local. Esta logró adaptarse a las condiciones de la zona manteniendo un alto rendimiento, sin embargo, por motivos de preferencias culturales al grano blanco, los productores indicaron no utilizarlo para consumo humano. En el campo se observó un alto grado de variabilidad en los caracteres morfológicos de la actual variedad amariIlo, que imposibilitó su agrupación como variedad, indicando que es una mezcla. Por lo tanto, puede deducirse el cruce entre el original maíz amarillo con variedades originarias de la zona, con el propósito de aumentar el tamaño de mazorca de las variedades locales.

Las variedades criollas que han sido cultivadas por más de tres generaciones son: el maicito, producto de selección y mejoramiento de sus caracteres productivos; el cuarenteño, producto de la presión y selección para recurso de emergencia en periodos de mucha sequía y el maíz negro, aunque este último no se siembra actualmente, solo por productores de mayor edad y con propósito de mantener la variedad.

No obstante que las variedades encontradas fueron descritas por los agricultores con base a una serie de características principalmente de la mazorca, como tamaño, forma, color de grano y altura de planta, los resultados de los análisis morfológicos reflejan una considerable variabilidad intravarietal.

Estos resultados concuerdan con la premisa de que existe un manejo tradicional de las variedades como un sistema abierto de material sembrado en la aldea El Trapiche, en el cual los campesinos promueven el flujo genético a través del intercambio y la mezcla de semillas y no aíslan, ni espacial ni temporalmente, las variedades cultivadas para evitar el intercambio de material genético. Por lo tanto, el continuo morfológico encontrado en este estudio respalda esta dinámica de conservación. Sin embargo, se requiere su confirmación a través de una evaluación estandarizada en una parcela experimental con condiciones ambientales homogéneas y una evaluación molecular para una identificación más certera y la determinación de las relaciones establecidas entre variedades.

La variedad maicito fue ampliamente caracterizada, al ser la más frecuente en las parcelas muestreadas. Tal como lo propone Louette (1996), esta es una variedad 
local, ya que su semilla ha sido reproducida en la región desde hace por lo menos 100 años. Dados los resultados obtenidos, en cuanto a la caracterización morfológica, su amplia utilización, así como su adaptación al clima seco de la región sur de Honduras, indica que el maicito tiene un enorme potencial de mejoramiento.

\section{AGRADECIMIENTO}

A los agricultores de la aldea El Trapiche por habernos dado la oportunidad de trabajar con su germoplasma, a los estudiantes de Biología por su colaboración en el estudio y a la Dirección de Investigación Científica y Posgrado (DICYP) de la UNAH por el financiamiento a este proyecto. Igualmente, a Thelma María Mejía Ordoñez por la revisión y correcciones a este manuscrito.

\section{BIBLIOGRAFÍA}

Fernández, L. y otros. (2000). Identificación y caracterización de razas de maíz en sistemas campesinos tradicionales de dos áreas rurales de Cuba. Revista Biociencias, 1(1), 3-17.

Ferro, E. y otros. (2008). Entendiendo el sistema informal de semilla de maíz cubano. La selección de variedades de maíz (Zea mays lin.) por campesinos de la palma, Pinar del Río. Cultura Tropical, 29(1), 61-68.

Hernández, X. E. (1985). Biología agrícola. Los conocimientos biológicos y su aplicación a la agricultura. México: CECSA.

Hernández, X. E. (1993). Aspects of plant domestication in México. A personal view. In Ramamoorthy, T. P.; Bye, R. J.; Lot, A. \& Fa, J. (Eds). Biological diversity of México: Origins and distribution. NY: Oxford University Press.

Jarvis, D. I.; Hodgkin, T. (1999). Wild relatives and crop cultivars: detecting natural introgression and farmer selection of new genetic combinations in agroecosystems. Molecular Ecology, 8(1), S159-S173.

Louette, D. (1996). Intercambio de semillas entre agricultores y flujo genético entre variedades de maíz en sistemas agrícolas tradicionales. En Serratos, J. A.; Willcox, M.C. y Castillo, F. (Eds.). Flujo genético entre maíz criollo, maíz mejorado y teocintle: implicaciones para el maíz transgénico. México: CIMMYT. 
Simons, C.; Tarano, J.M. and Pinto, J.H. (1959). Clasificación de reconocimiento de los suelos de la República de Guatemala. Guatemala: Instituto Agropecuario Nacional.

Toledo, V. M. (1990). The ecological rationality of peasant production. In Altieri, M. and Hecht, S. (Eds.). Agroecology and small-farm development. Florida: CRC Press. Tuxill, J. y Nabhan, G.P. (2001). Plantas, comunidades y áreas protegidas, una guía para el manejo in situ. Uruguay: Nordan-Comunidad. 